Ciência Florestal, Santa Maria, v. 23, n. 3, p. 337-344, jul.-set., 2013

ISSN 0103-9954

\title{
CARACTERIZAÇÃO DA FAUNA EDÁFICA EM PLANTIOS DE Eucalyptus spp.
}

\author{
SOIL FAUNA CHARACTERIZATION IN Eucalyptus spp. PLANTATIONS
}

\author{
Juliana Garlet ${ }^{1}$ Ervandil Correa Costa ${ }^{2}$ Jardel Boscardin $^{3}$
}

\begin{abstract}
RESUMO
Solos florestais propiciam boas condições para o desenvolvimento e estabelecimento da fauna edáfica, devido principalmente à deposição de serrapilheira. No entanto, sistemas conduzidos em monoculturas por fornecerem um único substrato alimentar, podem propiciar o desenvolvimento de determinados grupos faunísticos em detrimento de outros, ocasionando surtos de espécies-praga. O objetivo deste trabalho foi caracterizar a fauna edáfica e sua relação com as variáveis meteorológicas, em plantios de Eucalyptus spp. em Alegrete e São Francisco de Assis, Rio Grande do Sul. Este estudo foi conduzido em seis talhões de Eucalyptus, pertencentes a três espécies: Eucalyptus dunnii Maiden, Eucalyptus grandis Maiden e Eucalyptus grandis x Eucalyptus urophylla S. T. Blake (clone híbrido) e duas idades (plantio em 2006 e 2007). Os talhões estavam localizados nas fazendas Cabanha da Prata e Chica Barbosa município de Alegrete, e Taquari município de São Francisco de Assis, Rio Grande do Sul. Para a amostragem da fauna edáfica, foram utilizadas armadilhas de solo, sendo instaladas oito armadilhas distantes dez metros uma da outra, distribuídas em duas linhas de plantio no centro de cada talhão, totalizando 48 armadilhas. As coletas foram realizadas quinzenalmente, de setembro de 2008 a agosto de 2009. Após contagem e identificação dos grupos taxonômicos, foram determinados os parâmetros de Riqueza, Abundancia e Índice de Diversidade de Shannon. Os parâmetros determinados foram analisados segundo modelo fatorial 3 x 2 (três espécies e duas idades) em delineamento inteiramente casualizado pelo teste F. Durante este levantamento foram coletados 8.811 espécimes distribuídos em 12 grupos taxonômicos, sendo que os grupos, Coleoptera, Hymenoptera e Diptera apresentaram as maiores porcentagens de indivíduos coletados: 31,1, 23,2 e 19,6\%, respectivamente. Os plantios com três anos de idade apresentaram maior número de indivíduos coletados, quando comparados com os talhões de dois anos de idade. As espécies Eucalyptus grandis $x$ Eucalyptus urophylla e Eucalyptus grandis apresentaram um número superior de indivíduos coletados, quando comparados com a espécie Eucalyptus dunnii, nas duas idades avaliadas. Assim, a espécie e a idade do plantio exerceram influência sobre os organismos coletados.
\end{abstract}

Palavras-chave: entomologia florestal; organismos de solo; plantios florestais.

\section{ABSTRACT}

Forest soils provide good conditions for the development and the establishment of soil fauna, manly by the deposition of litter. However, monoculture systems conducted in a single food providing substrate, can promote the development of certain fauna groups over others, causing outbreaks of pest species. The aim of this study was to characterize the soil fauna and its relationship with meteorological variables, in plantations of Eucalyptus spp. This study was conducted in six stands of three species of Eucalyptus: Eucalyptus dunni Maiden, Eucalyptus grandis Maiden and Eucalyptus grandis x Eucalyptus urophylla S. T. Blake (clone hybrid) and two ages (planted in 2006 and 2007). The stands was located at Cabanha da

1 Doutora em Engenharia Florestal pelo Programa de Pós-graduação em Engenharia Florestal, Universidade Federal de Santa Maria. Professora do Curso de Engenharia Florestal da Universidade do oeste de Santa Catarina. Rua Dirceu Giordani, 696, CEP:89820-000, Xanxerê (SC). julianagarlet@yahoo.com

2 Engenheiro Agrônomo, Dr., Professor do Programa de Pós-Graduação em Engenharia Florestal. Centro de Ciências Rurais, Universidade Federal de Santa Maria, Av. Roraima, 1000, CEP 97105-900, Santa Maria (RS).ervandilc@gmail.com

3 Engenheiro Florestal, Ms., Doutorando do Programa de Pós-graduação em Engenharia Florestal, Centro de Ciências Rurais, Universidade Federal de Santa Maria, Av. Roraima, 1000, CEP 97105-900, Santa Maria (RS). Bolsista Capes. boscardinj@gmail.com

Recebido para publicação em 4/06/2009 e aceito em 23/04/2012

Ci. Fl., v. 23, n. 3, jul.-set., 2013 
Prata and Chica Barbosa farms in Alegrete, and at Taquari farm in São Francisco de Assis, Rio Grande do Sul state. For sampling soil insects, pitfall traps were used. Eight traps were installed ten meters apart from each other, distributed in two rows in the center of each stand, totaling 48 traps. Samples were collected fortnightly from September 2008 to August 2009. After counting and identifying the taxonomic groups, the parameters of richness (total number of taxonomic groups), abundance and shannon diversity index were determined. The results were analyzed using $3 \times 2$ factorial model (three species and two ages) in a randomized design with Test F. During this study, 8.811 specimens representing 12 taxonomic groups were collected. The groups Coleoptera, Hymenoptera, and Diptera had the highest percentages of individuals collected: $31.1,23.2$, and $19.6 \%$, respectively. The plantation with three years of age had a higher number of individuals compared to the stands of two years of age. The species Eucalyptus grandis and Eucalyptus grandis $x$ Eucalyptus urophylla had a higher number of individuals collected when compared with the species Eucalyptus dunnii at both ages. Thus, the species and age of the planting exerted influence on the organisms collected.

Keywords: forest entomology; soil organisms; forest plantations.

\section{INTRODUÇÃO}

A fauna do solo é construída de micro-organismos e invertebrados responsáveis pelo processo de decomposição e ciclagem de nutrientes (CORREIA e OLIVEIRA, 2000). Estes organismos favorecem mudanças nas propriedades físicas, químicas e biológicas do solo, onde o sistema solo-serrapilheira constitui habitat para uma variedade de organismos da fauna edáfica, embora seja bastante complexa do ponto de vista qualitativo e quantitativo (PERRANDO, 2008).

Diferentes coberturas vegetais e práticas culturais tendem a atuar diretamente sobre a população da fauna edáfica. Isso se deve principalmente à permanência de resíduos orgânicos sobre a superfície do solo. Geralmente coberturas com camada espessa de folhas mortas e com vários estratos de matéria fresca e em decomposição são capazes de abrigar uma fauna mais diversificada (CANTO, 1996). A diversidade de espécies da fauna edáfica está relacionada com a riqueza de espécies (número de espécies) e a distribuição do número de indivíduos entre as espécies (WALKER, 1989). Essa definição pode ser evidenciada no Índice de Shannon, que agrega essas duas variáveis (ODUM, 1983). Connell (1978) destaca que, quando se avalia a complexidade de uma comunidade num sentido mais amplo, a própria riqueza de espécies pode ser utilizada como uma medida geral da diversidade.

Correia et al. (1999) classificam a fauna do solo com base em aspectos funcionais, agrupando-os em guildas. Os saprófagos que se alimentam diretamente dos resíduos de plantas, fragmentando-os, pertencem aos grupos: Blattodea, Dermaptera, Diplopoda, Diplura, Isopoda, Psocoptera e Symphyla.
Os predadores que se alimentam de outros organismos (Araneae, Chilopoda, Pseudoscorpionida e Hymenoptera), as larvas de insetos (larvas de Diptera, Coleoptera, Lepidoptera e Neuroptera), os grupos Coleoptera, Collembola e Thysanoptera e os insetos sociais (Formicidae e Isoptera) podem ser tanto saprófagos como predadores. Já os grupos Diptera, Homoptera, Heteroptera e Trichoptera são classificados como não edáficos, pois não apresentam funcionalidade conhecida. Devido à grande complexidade, tanto quantitativa quanto qualitativa dos organismos edáficos, o conhecimento de toda comunidade torna-se inviável, sendo possível retratar parcelas desta comunidade, com a determinação de grupos taxonômicos, fornecendo uma primeira inferência sobre a funcionalidade destes organismos no solo (STORK e EGGLETON, 1992, GONZÁLEZ et al., 1996).

Solos florestais propiciam boas condições para o desenvolvimento e estabelecimento da fauna edáfica, principalmente pela deposição de serrapilheira, onde os grupos edáficos comumente encontrados em florestas naturais são: Hymenoptera, Collembola, Coleoptera e Isoptera (MOÇO et al., 2005; FERREIRA e MARQUES, 1998; SILVA et al., 2011). Ferreira e Marques (1998) observaram número reduzido de grupos edáficos em plantio de eucalipto quando comparado com floresta nativa. Baretta et al. (2003) consideram que monocultivos tendem a favorecer determinados grupos faunísticos pelo fornecimento de um único substrato alimentar, podendo ocasionar o surgimento de insetos-praga. Até o momento, há poucos grupos edáficos considerados insetos-praga florestais importantes destacando-se os grupos Isoptera e algumas larvas de coleópteros, que podem ocasionar danos em mudas no início do estabelecimento dos povoamentos. 
Assim, o objetivo deste estudo foi caracterizar a fauna edáfica e sua relação com as variáveis meteorológicas, em plantios de Eucalyptus dunnii, Eucalyptus grandis e Eucalyptus grandis $\mathrm{x}$ Eucalyptus urophylla (clone híbrido) em duas idades (plantio em 2006 e 2007), em Alegrete e São Francisco de Assis, Rio Grande do Sul.

\section{MATERIAIS E MÉTODOS}

\section{Caracterização da área}

O estudo foi conduzido em seis talhões de Eucalyptus, com duas idades, pertencentes às espécies: Eucalyptus dunnii Maiden (talhão com 18 ha plantio em 2006, e 25 ha plantio em 2007), Eucalyptus grandis Maiden (talhão com 20 ha plantio em 2006, e 24 ha plantio em 2007) e clone híbrido Eucalyptus grandis x Eucalyptus urophylla S. T. Blake (talhão com 30 ha plantio em 2006, e 22 ha plantio em 2007). Os talhões com as espécies Eucalyptus dunnii e Eucalyptus grandis possuem espaçamento de plantio de 3 x 2 (três metros entre linhas e dois metros entre plantas), e o talhão com Eucalyptus grandis x Eucalyptus urophylla 3,5 x 2,5 (três metros e meio entre linhas e dois metros e meio entre plantas). Os talhões estão localizados nas fazendas Cabanha da Prata $\left(29^{\circ} 48^{\prime} 19^{\prime \prime} \mathrm{S}\right.$;

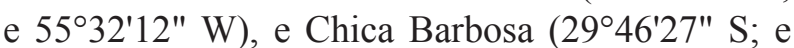
$55^{\circ} 34^{\prime} 37^{\prime \prime} \mathrm{W}$ ), (talhões com dois anos, plantio em 2007) município de Alegrete, e Taquari (29³3'38" S; e $55^{\circ} 17^{\prime} 10^{\prime \prime} \mathrm{W}$ ), (talhões com três anos, plantio em 2006) município de São Francisco de Assis, Rio Grande do Sul.

De acordo com a classificação de Köppen, o clima da região é denominado Cfah "Subtropical Mesotérmico" constantemente úmido, caracterizado por meses de frio, com geadas de maio a agosto, e calor intenso, principalmente nos meses de janeiro e fevereiro, com temperatura média do mês mais quente $>22^{\circ} \mathrm{C}$ e temperatura média anual $>18^{\circ} \mathrm{C}$ (MORENO, 1961). A precipitação geralmente apresenta-se bem distribuída durante todo ano, variando de $1.250 \mathrm{~mm}$ a $1.500 \mathrm{~mm}$, sem estação seca defini$\mathrm{da}$, com ventos dominantes de Sudoeste no inverno e Nordeste na primavera.

\section{Levantamento e coleta da fauna edáfica}

Para amostragem da fauna de solo foram instaladas armadilhas de interceptação (Pitfall), compostas de um recipiente cilíndrico de $10 \mathrm{~cm}$ de altura e $12 \mathrm{~cm}$ de diâmetro, sendo a área de captura de $38,5 \mathrm{~cm}^{2}$. As armadilhas foram mantidas enterradas de forma que sua abertura ficasse exatamente ao nível da superfície do solo, contendo apenas líquido conservante, constituído de uma solução de água e sal misturada com detergente $(250 \mathrm{~mL}$ de água + $20 \mathrm{~g}$ de sal +4 gotas de detergente). Foram instaladas oito armadilhas distantes dez metros uma da outra, distribuídas em duas linhas de plantio no centro de cada talhão, totalizando 48 armadilhas, sendo que estas eram retiradas no momento da coleta e instaladas novamente em novo local dentro das mesmas linhas de plantio avaliadas para a coleta posterior. As coletas foram realizadas quinzenalmente de setembro de 2008 a agosto de 2009.

Os indivíduos coletados foram triados no Laboratório de Entomologia Florestal do Departamento de Defesa Fitossanitária da Universidade Federal de Santa Maria, onde se realizou a contagem e identificação dos grupos taxonômicos. O reconhecimento dos grupos foi realizado com o auxílio de materiais bibliográficos (RUPPERT e BARNES, 1996; MORSELLI, 2009). Posteriormente, foram determinados os parâmetros de Riqueza (número total de grupos taxonômicos), Abundância e Índice de Diversidade de Shannon $(H)$. O Índice de Diversidade de Shannon foi obtido pela relação $(H=-\Sigma p i \cdot \log p i)$, onde: $p i=n i / N$; $n i=$ densidade de cada grupo, $N=$ número total de grupos, sendo calculado pelo programa estatístico Past (HAMMER et al., 2001).

Os parâmetros determinados foram analisados segundo modelo fatorial $3 \times 2$ (três espécies e duas idades) em delineamento inteiramente casualizado com oito repetições pelo teste F. Para os efeitos avaliados (espécie e idade) aplicou-se o teste de Tukey a 5\% de significância, utilizando o programa estatístico Assistat (SILVA e AZEVEDO, 2009).

\section{Dados meteorológicos}

Os elementos meteorológicos registrados foram: temperaturas médias, máximas e mínimas, precipitação e umidade relativa. As leituras foram diárias, no entanto, na apresentação dos resultados foram considerados os valores médios dos intervalos entre cada coleta. Os dados foram obtidos junto à estação meteorológica do Instituto Federal Farroupilha, Campus Alegrete (29071'16" S e $\left.55^{\circ} 52^{\prime} 61^{\prime \prime} \mathrm{W}\right)$. Com os dados das variáveis meteorológicas obtidas, verificou-se se havia correlação entre o número de insetos coletados e os elementos 
meteorológicos, utilizando-se a correlação linear calculada através do programa estatístico Assistat (SILVA e AZEVEDO, 2009).

\section{RESULTADOS E DISCUSSÕES}

Foram coletados 8.811 espécimes distribuídos em nove grupos taxonômicos, apresentados na Tabela 1.

As ordens Coleoptera, Hymenoptera, e Diptera apresentaram as maiores porcentagens de indivíduos coletados respectivamente, $31,1 \%$, $23,2 \%$ e 19,6 \% (Tabela 1). Em estudo realizado por Soares (1999), avaliando a fauna de solo em plantio de Eucalyptus sp., o autor constatou que as ordens Coleoptera, Hymenoptera, e Diptera foram as mais abundantes com $11,6,54,7$ e $11,6 \%$ dos organismos coletados respectivamente. A ordem Hymenoptera apresentou o maior número de indivíduos coletados, diferentemente do que ocorreu neste estudo onde a ordem Coleoptera foi a mais representativa. Com relação às espécies de Eucalyptus avaliadas, os plantios com três anos de idade apresentaram maior número de indivíduos coletados quando comparados com os talhões de dois anos de idade. E as espécies Eucalyptus grandis $x$ Eucalyptus urophylla e Eucalyptus grandis favoreceram a presença de um número superior de indivíduos em relação à espécie Eucalyptus dunnii, nas duas idades avaliadas.

A interação entre as espécies de Eucalyptus e as idades dos talhões foi significativa somente para o Índice de Diversidade de Shannon, para os parâmetros Abundância de insetos coletados e Riqueza, a interação não foi significativa (Tabela 2).

Perrando (2008) avaliando fauna de solo em plantio de Acacia mearnsii De wild, (com um ano de idade) encontrou a riqueza variando de oito (inverno) a onze (verão outono) de grupos taxonômicos. Moço et al. (2005), avaliando a fauna edáfica em plantio de Corymbia ciriodora com 18 anos, observaram valores médios máximos para a riqueza de 7,8. Neste estudo o número de grupos taxonômicos foi superior ao encontrado por Moço et al. (2005), e semelhante ao encontrado por Perrando (2008) (Tabela 2). A semelhança destes resultados com os obtidos por Perrando em monocultivo de acácia (2008) pode estar associada ao fato de que os estudos foram desenvolvidos na mesma região, tendo assim as mesmas características climáticas e edáficas.

TABELA 1: Grupos taxonômicos coletados em armadilhas de solo, em plantios de Eucalyptus spp., em São Francisco de Assis e Alegrete, RS (2008/2009).

TABLE 1: Taxonomic groups collected in pitfall traps in stands of Eucalyptus spp. in São Francisco de Assis and Alegrete, Rio Grande do Sul state (2008/2009).

\begin{tabular}{ccccccc}
\hline $\begin{array}{c}\text { Grupos } \\
\text { taxonômicos }\end{array}$ & $\begin{array}{c}\text { Eucalyptus } \\
\text { grandis } x \\
\text { Eucalyptus } \\
\text { urophylla } 06\end{array}$ & $\begin{array}{c}\text { Eucalyptus } \\
\text { grandis } x \\
\text { Eucalyptus } \\
\text { urophylla } 07\end{array}$ & $\begin{array}{c}\text { Eucalyptus } \\
\text { dunnii 06 }\end{array}$ & $\begin{array}{c}\text { Eucalyptus } \\
\text { dunnii 07 }\end{array}$ & $\begin{array}{c}\text { Eucalyptus } \\
\text { grandis 06 }\end{array}$ & $\begin{array}{c}\text { Eucalyptus } \\
\text { grandis 07 }\end{array}$ \\
\hline Arachnida $^{1}$ & $86^{3}$ & 48 & 60 & 73 & 76 & 118 \\
Blattodea & 2 & 2 & 3 & 8 & 1 & 0 \\
Collembola & 116 & 54 & 73 & 129 & 238 & 102 \\
Coleoptera & 447 & 579 & 593 & 285 & 719 & 383 \\
Crustacea & 16 & 0 & 3 & 0 & 3 & 2 \\
Diptera & 574 & 195 & 195 & 260 & 334 & 338 \\
Hemiptera & 50 & 9 & 28 & 27 & 30 & 20 \\
Hymenoptera & 480 & 365 & 312 & 300 & 419 & 365 \\
Formas imaturas & 90 & 40 & 60 & 63 & 68 & 42 \\
Lepidoptera & 5 & 3 & 2 & 5 & 0 & 2 \\
Opiliones & 70 & 35 & 37 & 47 & 120 & 64 \\
Orthoptera & 150 & 68 & 25 & 35 & 70 & 48 \\
\hline$\sum$ & 2.086 & 1.398 & 1.391 & 1.232 & 2.078 & 1.484 \\
\hline
\end{tabular}

Em que: ${ }^{1}=$ Grupo Arachnida composto por aranhas e escorpiões; ${ }^{2}=$ Formas imaturas espécimes coletados que se encontravam em fase de larva; ${ }^{3}=$ indivíduos coletados. 06-Plantio em 2006, 07- Plantio em 2007. 
TABELA 2: Médias de Abundância, Riqueza e Índice de Diversidade de Shannon de organismos coletados quinzenalmente em armadilhas de solo em plantios de Eucalyptus spp., em São Francisco de Assis e Alegrete, RS (2008/2009).

TABLE 2: Mean Abundance, Richness and Shannon Diversity Index of organisms collected fortnightly in pitfall traps in plantations of Eucalyptus spp. in São Francisco de Assis and Alegrete, Rio grande do Sul (2008/2009).

\begin{tabular}{llll}
\hline \multirow{2}{*}{ Espécies } & \multicolumn{3}{l}{ Abundância } \\
\cline { 2 - 4 } & Médias & Ano de Plantio & Médias \\
\hline Eucalyptus grandis $x$ Eucalyptus urophylla & $221,56 \mathrm{a}$ & 2006 & $235,33 \mathrm{a}$ \\
Eucalyptus dunnii & $177,25 \mathrm{~b}$ & 2007 & $186,87 \mathrm{~b}$ \\
Eucalyptus grandis & $234,5 \mathrm{a}$ & - & - \\
\hline CV \% & 16,6 & - & - \\
\hline & \multicolumn{3}{c}{ Riqueza } \\
\cline { 2 - 4 } & Médias & Ano de Plantio & Médias \\
\cline { 2 - 4 } Eucalyptus grandis $x$ Eucalyptus urophylla & $9,56 \mathrm{a}$ & 2006 & $9,5 \mathrm{a}$ \\
Eucalyptus dunnii & $9,68 \mathrm{a}$ & 2007 & $9,45 \mathrm{a}$ \\
Eucalyptus grandis & $9,19 \mathrm{a}$ & - & - \\
\hline CV \% & 9 & - & - \\
\hline & & Índice de Diversidade de Shannon \\
\cline { 2 - 4 } & 2006 & & 2007 \\
Eucalyptus grandis $x$ Eucalyptus urophylla & $1,83 \mathrm{Aa}$ & & $1,57 \mathrm{Bb}$ \\
Eucalyptus dunnii & $1,58 \mathrm{Bb}$ & & $1,79 \mathrm{Aa}$ \\
Eucalyptus grandis & $1,76 \mathrm{Aa}$ & & - \\
\hline CV\% & 6,27 & & $1,73 \mathrm{Aa}$ \\
\hline
\end{tabular}

Médias seguidas pela mesma letra não diferem estatisticamente pelo teste de Tukey a 5\% de significância.

Antoniolli et al. (2006), estudando a fauna em solo em plantio de Eucalyptus sp. com dois e seis anos de idade, em São Francisco de Assis, $\mathrm{RS}$, observaram valores médios para Riqueza de 7,6 para o povoamento com dois anos e de oito para o povoamento com seis anos. Para a Abundância os autores observaram valores de 104 e 176 para os povoamentos com dois e seis anos respectivamente. Observou-se neste estudo que, apesar dos povoamentos serem recentes (dois e três anos), os valores médios de Riqueza e Abundância (Tabela 2) foram superiores aos encontrados por Antoniolli et al. (2006), na mesma região, demonstrando que as áreas avaliadas neste estudo apresentam boas condições para o estabelecimento de fauna edáfica.

Analisando-se os Índices de Diversidade de Shannon (Tabela 2), observa-se que para a espécie Eucalyptus grandis, o Índice de Diversidade foi semelhante para as duas idades de plantio, enquanto que para Eucalyptus grandis $x$ Eucalyptus urophylla, o plantio com três anos apresentou índice superior ao de dois anos de idade. Para Eucalyptus dunnii, ocorreu o inverso o plantio com dois anos obteve maior índice de diversidade que o com três anos. Normalmente, espera-se que, com o aumento da idade do povoamento os índices de diversidade sejam superiores devido ao aumento da matéria orgânica pela deposição de serrapilheira, propiciando melhores condições para o desenvolvimento da meso e macro fauna do solo. Segundo Ward e Rogers (2007), atributos como textura, conteúdo de água no solo, matéria orgânica, e características químicas podem influenciar muitas das características comportamentais das espécies edafícolas.

Moço et al. (2005), estudando a fauna edáfica em plantio de Corymbia ciriodora, obtiveram um Índice de Shannon máximo de 2,6 (no verão), superior ao encontrado neste estudo, isto pode ser explicado pelo fato de que povoamento avaliado por Moço et al. (2005), apresenta idade significativamente superior (18 anos), aos povoamentos avaliados neste estudo (dois e três anos), propiciando assim melhores condições para o desenvolvimento e estabelecimento de organismos edáficos.

Com base nos totais observados, os grupos taxonômicos distribuem-se de maneira diferente ao longo das estações do ano (Figura 1). Os grupos 


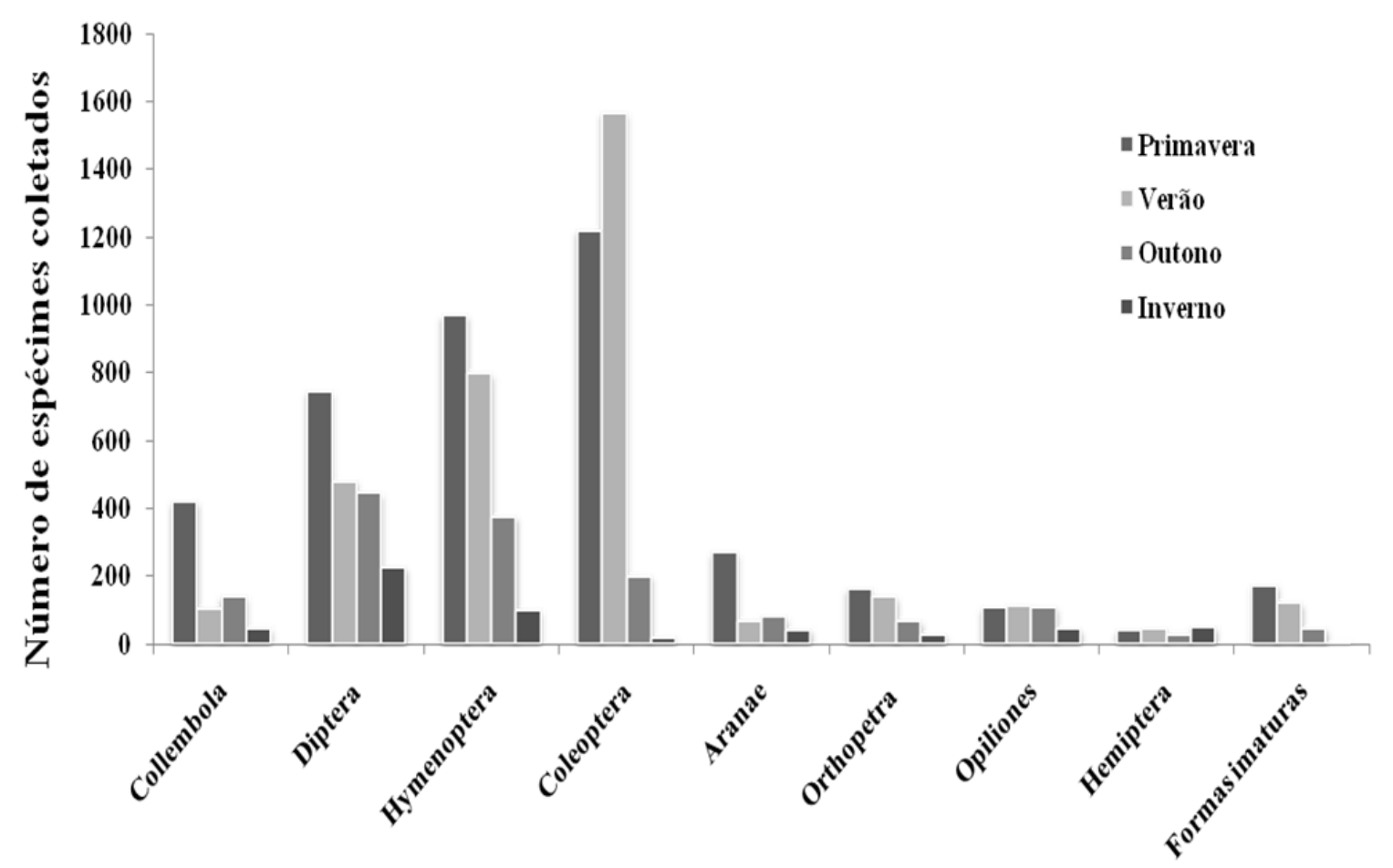

Grupos taxonômicos

FIGURA 1: Distribuição da meso e macro fauna edáfica ao longo das estações do ano em plantios de Eucalyptus spp., em São Francisco de Assis e Alegrete, RS (2008/2009).

FIGURE 1: Distribution of meso- and macro-soil fauna throughout the seasons in stands of Eucalyptus spp. in São Francisco de Assis and Alegrete, Rio Grande do Sul state (2008/2009).

Diptera, Hymenoptera, Orthoptera e Formas imaturas foram encontrados em maior número na primavera, decrescendo no verão, outono e inverno. Collembola e Aranae foram coletados em maior quantidade na primavera, reduzindo significativamente nas demais estações. Coleópteros foram capturados em maior quantidade no verão e na primavera respectivamente, e os grupos Opiliones e Hemiptera apresentaram distribuição semelhante ao longo das estações. A fim de melhor compreender as relações entre os grupos taxonômicos coletados e sua distribuição ao longo das estações do ano, realizou-se a correlação linear entre o número de organismos coletados e as principais variáveis meteorológicas (Tabela 5).

A maioria dos grupos taxonômicos encontrados neste estudo apresentou relação com as variáveis meteorológicas temperatura e umidade relativa (Tabela 3). Os grupos Coleoptera e Orthoptera apresentaram os maiores valores de relação com temperatura e umidade relativa. Segundo Teixeira et al. (2009), em levantamentos de coleópteros de solo, geralmente observa-se que estes são mais abundantes no período de maiores temperaturas. Para Sydow et al. (2007), este fato pode estar relacionado à oferta de recursos ser máxima nos meses quentes e mínima nos meses frios, assim a distribuição da fauna se adapta a estas variações, aumentando sua população com a elevação da temperatura.

Perrando (2008), ao estudar a fauna epiedáfica em plantios de Acacia mearnsii, no Rio Grande do Sul, verificou alta correlação (> 0,7) entre os grupos Hymenoptera e Orthoptera com a temperatura média do período, enquanto Collembola apresentou correlação somente com a precipitação. Neste estudo, ortópteros também apresentaram alta correlação com a temperatura média e para himenópteros a correlação com a temperatura foi moderada $(>0,5)$. Já os colêmbolos não apresentaram correlação com nenhuma das variáveis meteorológicas estudadas. Em estudos realizados por Rovedder et al. (2004) e Vitti et al. (2004), os autores observaram que a redução da precipitação pluvial provocou a diminuição do número de organismos do solo, principalmente no grupo Collembola, a não correlação deste grupo com as variáveis ambientais, pode estar relacionada ao método de coleta, que não se mostrou eficiente para este grupo. 
TABELA 3: Tabela de relação entre o número médio de indivíduos dos grupos taxonômicos coletados quinzenalmente em armadilhas de solo e as principais variáveis meteorológicas, em plantios de Eucalyptus spp., em São Francisco de Assis e Alegrete, RS (2008/2009).

TABLE 3: Table of linear relation between the average individual numbers of taxonomic groups collected fortnightly in pitfall traps and the main meteorological variables in plantations of Eucalyptus spp. São Francisco de Assis and Alegrete, Rio Grande do Sul (2008/2009).

\begin{tabular}{cccccc}
\hline Grupos taxonômicos & $\mathrm{PP}(\mathrm{mm})$ & T. máx C & T. min $\mathrm{C}^{\circ}$ & T. média C & U.R. \% \\
\hline Arachnida $^{1}$ & 0,1662 & $-0,2602$ & $-0,2526$ & $-0,2206$ & 0,1909 \\
Blattodea & $-0,3421$ & $0,5714^{*}$ & $0,5616^{*}$ & $0,5570^{*}$ & $0,6696^{*}$ \\
Collembola & 0,4094 & 0,0402 & 0,0536 & 0,0590 & 0,0828 \\
Coleoptera & $-0,0565$ & $0,7567^{*}$ & $0,7559^{*}$ & $0,7558^{*}$ & $0,7235^{*}$ \\
Crustacea & 0,5729 & 0,3669 & 0,3764 & 0,3298 & $-0,1921$ \\
Diptera & 0,0154 & $0,6021^{*}$ & $0,6006^{*}$ & $0,5828^{*}$ & $0,6002^{*}$ \\
Hemiptera & $-0,2300$ & $-0,115$ & $-0,1294$ & $-0,1920$ & $-0,3747$ \\
Hymenoptera & $-0,1903$ & $0,5582^{*}$ & $0,5550^{*}$ & $0,5614^{*}$ & $0,5981^{*}$ \\
Formas imaturas ${ }^{2}$ & 0,3201 & $0,6525^{*}$ & $0,6559^{*}$ & $0,6514^{*}$ & $0,5334^{*}$ \\
Lepidoptera & $-0,3890$ & $0,5095^{*}$ & 0,4938 & 0,4702 & $0,5587^{*}$ \\
Opiliones & $-0,2771$ & $0,6633^{*}$ & $0,6511^{*}$ & $0,6481^{*}$ & $0,6363^{*}$ \\
Orthoptera & $-0,0219$ & $0,7505^{*}$ & $0,7491^{*}$ & $0,7539^{*}$ & $0,6708^{*}$ \\
\hline
\end{tabular}

Em que: ${ }^{1}=$ Grupo Arachnida composto por aranhas e escorpiões; ${ }^{2}=$ Formas imaturas espécimes coletados que se encontravam em fase de larva; $\mathrm{PP}=$ precipitação; $\mathrm{T}$ máx $\mathrm{C}^{\circ}=$ temperatura máxima; $\mathrm{T}$ min $\mathrm{C}^{\circ}=$ temperatura mínima; T média $\mathrm{C}^{\circ}=$ temperatura média; $\mathrm{UR}=$ umidade relativa; ${ }^{* *}$ significativo ao nível de $1 \%$ de probabilidade $(\mathrm{p}<0.01)$, * significativo ao nível de $5 \%$ de probabilidade $(01=<\mathrm{p}<0.05)$, ns não significativo ( $\mathrm{p}>=0.05)$.

\section{CONCLUSÕES}

- Na área em estudo, observaram-se as ordens Coleoptera, Hymenoptera e Diptera como as mais abundantes;

- As espécies Eucalyptus grandis $x$ Eucalyptus urophylla e Eucalyptus grandis apresentaram número superior de indivíduos em relação à espécie Eucalyptus dunnii, nas duas idades avaliadas, e nos plantios com três anos observou-se maior número de indivíduos coletados;

- As variáveis meteorológicas temperatura e umidade relativa exerceram significativa influência no número de espécimes coletados nos diferentes grupos taxonômicos.

\section{AGRADECIMENTOS}

Ao Conselho Nacional de Desenvolvimento Cientifico e Tecnológico (CNPq), pela concessão da bolsa de Mestrado da primeira autora. E à empresa StoraEnso Florestal pelo apoio logístico na execução das coletas.

\section{REFERÊNCIAS BIBLIOGRÁFICAS}

ANTONIOLLI, Z. I. et al. Método alternativo para estudar a fauna do solo. Ciência Florestal, Santa Maria, v. 16, n. 4, p.407-417, out./dez. 2006.

BARETTA, D. et al.Fauna edáfica avaliada por armadilhas de catação manual efetada pelo manejo do solo na região oeste catarinense. Revista Ciência Agroveterinárias, Lages, v. 2, p. 97-106, jul./dez. 2003.

CANTO, A. C. Alterações da mesofauna do solo causadas pelo uso de cobertura com plantas leguminosas na Amazônia Central. Revista Ciências Agrárias, Recife, v. 4, n. 5, p. 79-94, out./ dez. 1996.

CONNELL, J. H. Diversity in tropical rain forests and coral reefs. Science, Washington, n. 199, p. 1302-1310, 1978.

CORREIA, M.E. F.; ANDRADE, A. G. Formação de serapilheira e ciclagem de nutrientes. In: SANTOS, G. A.; CAMARGO, F. A. O., eds. Fundamentos da matéria orgânica do solo: ecossistemas tropicais e subtropicais. 1. Porto Alegre, Genesis, 1999. p.197- 
225.

CORREIA, M. E. F.; OLIVEIRA, L. C. M. Fauna do solo: Aspectos Gerais e Metodológicos. Seropédica: Embrapa Agrobiologia, 2000. 46 p. FERREIRA, R. L; MARQUES, M. G. S. M. A Fauna de Artrópodes de Serrapilheira de Áreas de Monocultura com Eucalyptus sp. e Mata Secundária Heterogênea. Anais da Sociedade Entomológica do Brasil. Curitiba, v. 27, n. 3, p. 395-403. set./dez. 1998.

GONZÁLEZ, G.;ZOU, X.; BORGES, S. Earthworm abundance and species composition in abandoned tropical croplands: comparison of tree plantations and secondary forests. Pedobiologia, Berlin, v. 40, p. 385-391, 1996.

HAMMER, O.; HARPER, D. A. T.; RYAN, P. D. PAST: Paleontological Statistics Software Package for Education and Data Analysis. Palaeontologia Electronica v. 4, n.1, 2001. 9 p.

MOÇO, M. K da S. et al. Caracterização da fauna edáfica em Diferentes coberturas vegetais na Região norte fluminense. Revista Brasileira de Ciência do Solo, Viçosa, v. 29, n. 4, p. 555-564, jul./agt. 2005. MORENO, J. A. Clima do Rio Grande do Sul. Porto Alegre, Secretaria da Agricultura, 1961. 73 p. MORSELLI, T. B. G. A. Biologia do Solo. Pelotas, Editora e Gráfica Universitária PREC-UFPel, 2009. $145 \mathrm{p}$.

ODUM, E. P. Ecologia. Rio de Janeiro, Guanabara, 1983. $434 \mathrm{p}$.

PERRANDO, E. R. Caracterização física e biológica do solo após aplicação de herbicidas em plantios de Acácia-Negra (Acacia mearnsii De Wild.) no Rio Grande Do Sul. 2008. 93 f. Tese (Doutorado em Engenharia Florestal) Universidade federal de Santa Maria, Santa Maria, 2008.

ROVEDDER, A. P. et al. Fauna edáfica em solo susceptivel à arenização na região sudoeste do Rio Grande do Sul. Revista de Ciências Agroveterinárias, Lages, v. 3, n. 2, p. 87-96, jul./ dez. 2004.

RUPPERT, E. E.; BARNES, R. D. Zoologia dos invertebrados. São Paulo, Roca, 1996. 1074 p.
SILVA, A. P. Estudo da diversidade de arthropoda em fragmentos de mata no distrito de Rolim De Moura Do Guaporé - RO. Revista Eletrônica da Facimed, Cacoal, v. 3, n. 3, p. 315-321, jan/jul.2011. SILVA, F.DEA. S.; AZEVEDO, C.A. V.de. Principal Components Analysis in the Software AssistatStatistical Attendance. In: WORLD CONGRESS ON COMPUTERS IN AGRICULTUMIDADE RELATIVAE, 7., 2009, Reno. Anais...American Society of Agriculal and Biological Engineers, 2009.

SOARES, M. I. J. Meso e macrofauna do solo sob diferentes coberturas vegetais. 1999. $105 \mathrm{f}$. Dissertação (Mestrado em Engenharia Florestal) Universidade federal de Santa Maria, Santa Maria, 1999, 1999.

STORK, N. E.; EGGLETON, P. Invertebrates as determinants and indicators of soil quality. American Journal of Alternative Agriculture, Cambridge ,v. 7, p. 38-47, Jan./Dec. 1992.

SYDOW, V. G. et al. Aspectos estruturais da fauna de solo em áreas sob influência do processamento do carvão mineral no Sul do Brasil. In: Congresso de Ecologia do Brasil, 8., 2007, Caxambu. Anais ... Caxambu :Sociedade Brasileira de Ecologia, 2007. TEIXEIRA, C. C. L., HOFFMANN, M.; SILVAFILHO, G. Comunidade de Coleoptera de solo em remanescente de Mata Atlântica no estado do Rio de Janeiro, Brasil. Biota Neotropica, v. 9, n. 4. out./ dez. p. 91-96. 2009.

VITTI, M. R. et al. Estudo da mesofauna (ácaros e colêmbolos) em um pomar de pessegueiro conduzido sob uma perspectiva agroecológica. In: FERTBIO, 2004, Lages, Anais... Lages, SBCS, 2004.

WALKER, D. Diversity and stability. In: CHERRETT, J. M., ed. Ecological concepts. Oxford, Blackwell Scientific Public, 1989. p.115146.

WARD, A. L; ROGERS, D. J. Oviposition response of scarabaeids: does 'mother knows best' about rainfall variability and soil moisture? Physiological Entomology, London, v. 32, n. 4, p. 357-366, Sept./ Dec. 2007. 